\title{
COVID-19 vaccine uptake among health care workers in Ghana: a case for targeted vaccine deployment campaigns in the global south
}

\author{
Robert Kaba Alhassan* ${ }^{*}$, Seth Owusu-Agyei, Evelyn Korkor Ansah and Margaret Gyapong
}

\begin{abstract}
Background: Health care workers (HCWs) are among the high-risk groups in contracting and dying from COVID-19. World Health Organization estimates that over 10,000 HCWs in Africa have been infected with COVID-19 making it a significant occupational health hazard to HCWs. In Ghana, over 100 HCWs have already been infected and dozen others died from the virus. Acceptability and uptake of the COVID-19 vaccine is therefore critical to promote health and safety of HCWs as the country battles out of a third wave of the pandemic.

Objective: The study sought to ascertain the correlates of HCWs likelihood of participating in a COVID-19 vaccine trial and accepting the vaccine when given the opportunity.

Methods: The study was a web-based cross-sectional survey among HCWs $(n=1605)$ in all sixteen (16) administrative regions in Ghana. Data were analyzed with STATA statistical analysis software (version 14). Chi-square $\left(X^{2}\right)$ and Fisher's exact tests were used to test for differences in categorical variables; bivariate probit regression analysis with Average Marginal Effect (AME) was employed to ascertain the determinants of HCWs'likelihood of participating in a COVID-19 vaccine trial and taking the vaccine.
\end{abstract}

Results: It was found that $48 \%$ of HCWs will participate in a COVID-19 vaccine trial when given the opportunity; $70 \%$ will accept the COVID-19 vaccine; younger HCWs (AME $=0.28, \mathrm{SE}=0.16, p<0.1)$, non-Christians $(\mathrm{AME}=21, \mathrm{SE}=0.09$, $p<0.05$ ) and those who worked in faith-based health facilities ( $\mathrm{AME}=18, \mathrm{SE}=0.07, p<0.05$ ) were more likely to participate in a COVID-19 vaccine trial. Female HCWs $(A M E=-11, S E=0.04, p<0.05)$ and those with lower educational qualification were less likely to accept a COVID-19 vaccine (AME $=-0.16, \mathrm{SE}=0.08, p<0.1)$. Reasons cited for unwillingness to participate in a COVID-19 vaccine trial or uptake the vaccine were mainly fear, safety concerns, mistrust, uncertainty, spiritual and religious beliefs.

Conclusions: Acceptance of the COVID-19 vaccine appear to be high among HCWs; conversely, willingness to volunteer for the vaccine trial was low. Continuous targeted and integrated public health education for HCWs will enhance vaccine acceptability to promote safety and population health in the global south as Ghana intensifies efforts to produce COVID-19 vaccines locally.

Keywords: Coronavirus disease 2019 (COVID-19), Vaccine, Health care workers, Trial, Willingness, Uptake, Ghana

*Correspondence: arkabason@gmail.com; ralhassan@uhas.edu.gh Institute of Health Research (IHR), University of Health and Allied Sciences, PMB 31, Volta Region, Ho, Ghana

\section{Background}

As at August, 2021, over 200 million cases of the novel coronavirus disease 2019 (COVID-19) were recorded globally. Out of this figure over 4 million people unfortunately died [1]. Within the African region over 4 million original author(s) and the source, provide a link to the Creative Commons licence, and indicate if changes were made. The images or other third party material in this article are included in the article's Creative Commons licence, unless indicated otherwise in a credit line to the material. If material is not included in the article's Creative Commons licence and your intended use is not permitted by statutory regulation or exceeds the permitted use, you will need to obtain permission directly from the copyright holder. To view a copy of this licence, visit http://creativecommons.org/licenses/by/4.0/. The Creative Commons Public Domain Dedication waiver (http://creativeco mmons.org/publicdomain/zero/1.0/) applies to the data made available in this article, unless otherwise stated in a credit line to the data. 
cases have been recorded so far with over 100000 deaths [2]. In Ghana, more than 100000 cases of COVID-19 have been recorded with over 1000 deaths [3].

Public health response across the globe at the initial phase of the pandemic generally focused on non-pharmaceutical interventions (NPIs) such as frequent hand washing, physical/social distancing and the use of face masks in public spaces [2]. Albeit these NPIs remain essential in the fight against the global pandemic, effective vaccine deployment is an ultimate intervention to propel humanity back to normal lives. In the later part of 2020, breakthroughs were announced of different variants of COVID-19 vaccines which got World Health Organization (WHO) emergency use authorization (EUA) [4].

At the time of writing this paper, at least seven different COVID-19 vaccines on three platforms have been approved by the WHO [4] and over billion doses of the vaccine already administered globally as at 4th May, 2021. Nonetheless, this figure represents barely $7.9 \%$ of the global population receiving first dose and another $3.6 \%$ receiving second dose of the vaccine [4].

Africa has so far administered first dose of the COVID19 vaccine to barely $0.99 \%$ of its population and second dose administered to $0.35 \%$ of the continent's population as at May, 2021 [2]. Health care workers (HCWs) are among the high-risk groups in terms of contracting the virus and equally dying from it because of their closer contact with COVID-19 cases [5].

WHO African Regional Office estimates that over 10 $000 \mathrm{HCWs}$ in Africa have been infected with the disease $[6,7]$. In Ghana, anecdotal information suggests over 100 HCWs have already been infected and over 10 others died from the virus. To address this occupational health hazard, HCWs are among the prioritized category of persons earmarked for vaccination against the virus to promote their safety. Even though the vaccine does not give absolute protection from infection, available empirical data suggests it reduces risk of hospitalization and other severe outcomes including death [8-11].

Nonetheless, there are concerns of fear, anxiety and hesitancy among the populace including HCWs which threatens successful rollout of the vaccine in many countries including Ghana. Ghana like many African countries has had its first share of resistance to vaccine trials on account of fear, anxiety, lack of trust and conspiracy theories. In the year 2015, there was suspension of a planned Phase 1 trial for the Janssen Ebola vaccine at the University of Health and Allied Sciences (UHAS) campus in Hohoe, Volta region in Ghana, and Phase 2 trial for the GlaxoSmithKline (GSK) Ebola vaccine in Hohoe and Kintampo also in Ghana [12]. Similarly, in 2003 in northern Nigeria, polio vaccination campaigns were boycotted
[13] largely because of earlier experience related to an antibiotic trial (Trovan) in 1996 when 11 children were reported to have died, even though there was no evidence the deaths were due to the trial [13]. Recent malaria vaccine equally met some resistance at the early stages in Ghana which nearly halted the trial but was finally rolled out on 30th April 2019 [14].

Even though vaccine hesitancy, even among HCWs, remains an important threat to successful rollout of vaccines there are still limited scientific studies within the Ghanaian context to unearth the correlates of the phenomenon. Moreover, few studies have investigated into HCWs likelihood of accepting to participating in COVID-19 vaccine trial and uptake of the vaccine, when given the opportunity. Available studies, on COVID-19 vaccine uptake are either outside the Ghanaian context or conducted after deployment of the COVID-19 vaccine when citizens were already exposed to reactogenicity of the vaccine [15-22]. As Ghana intensifies in efforts towards producing the COVID-19 vaccines locally [23] and possibly conducting clinical trials on such vaccines, it is imperative the process is driven by empirical data on likelihood of stakeholders accepting to participate in these trials and uptake the vaccines.

This study therefore sought to examine the correlates of HCWs likelihood of participating in a COVID-19 vaccine trial and eventually uptake the vaccine when given the opportunity. The study was conducted between 18th September and 23rd October, 2020, before the first batch of COVAX facility of AstraZeneca vaccine was delivered in Ghana for the first time in the sub-region [24].

\section{Methods \\ Study design}

The study was a cross-sectional descriptive survey conducted prior to deployment of COVID-19 vaccine in Ghana. The study sought to ascertain frontline health workers' likelihood of participating in a COVID-19 vaccine trial and uptake the vaccine when given the opportunity.

\section{Study setting/population/sampling}

The study was conducted in all sixteen (16) administrative regions in Ghana targeting frontline HCWs at various levels of the healthcare delivery system. Ghana had over $115000 \mathrm{HCW}$ employed in the public sector as at 2018 , with nearly $60 \%$ being nurses and midwives [25], cited in Asamani et al. [26]. Unpublished data suggests the HCWs workforce has appreciated over the 2018 figure. Using Krejcie and Morgan's [27] formula for calculating sample size based on known population, the minimum required sample size of 384 at $95 \%$ confidence 
level was deemed representative. Nonetheless, a total of 1605 responses were received from the nationwide webbased survey, representing at least $10 \%$ coverage of the population of health workforce in Ghana.

\section{Instruments of data collection}

The study employed a web-based nationwide survey using RedCap software. Questionnaire administration was done via social media platforms and networks between 18th September and 23rd October, 2020 to eligible respondents who voluntarily consented to participate in the survey. Participants who could not access the webbased survey link were assisted to answer the questions by trained research assistants from all the 16 regions. The data collection instrument comprised of open and close ended questions. The questionnaire sections were on socio-demographic characteristics; experiences with COVID-19; views on COVID-19 vaccine trials and the vaccine, and likelihood of accepting vaccination when given the opportunity. Internal reliability of the questionnaire items was tested using Cronbach's alpha test and the average scale reliability coefficient was above the $80 \%$ rule of thumb.

\section{Ethical considerations}

The study got ethical clearance from the Research Ethics Committee (REC) of the University of Health and Allied Sciences, Ghana (Clearance Number: UHAS-REC A.1[6]20-21). Participation was voluntary and only participants who provided voluntary informed consent were included in the study. Privacy and confidentiality were assured through coding to anonymize personal information of respondents.

\section{Data analysis}

Data were analyzed with STATA statistical analysis software (version 14) after cleaning and coding. Main outcome variables of interest were whether or not respondents will participate in a COVID-19 vaccine trial (yes $=1$; no $=0)$ and accept or uptake a COVID-19 vaccine when given the opportunity (yes $=1$; no $=0$ ). Explanatory variables of interest were: $\operatorname{sex}($ male $=1$; female $=2)$; age (numeric); education (multiple response); professional category (multiple response); level of health facility where staff works (multiple response); region (multiple response); marital status (multiple response); religion (multiple response); willingness to pay for COVID-19 vaccine (yes $=1$, no $=2$ ) and whether or not staff works in a COVID-19 treatment centre (yes $=1$, no $=2$ ).

Chi-square $\left(X^{2}\right)$ and Fisher's exact tests were used to test for differences in categorical variables at $95 \%$ confidence level. Additionally, bivariate probit regression analysis using Average Marginal Effect (AME) estimations was employed to ascertain the determinants of HCW' readiness to participate in a COVID-19 vaccine trial and their likelihood of taking the vaccine when given the opportunity. The use of the bivariate probit regression is justified because of the dichotomous nature of the dependent variables [28]. Also, since the bivariate probit regression is a nonlinear model, its coefficients do not sufficiently inform on the magnitude of the effects of changes in the explanatory variables on an outcome variable [29]. In view of these strengths, AMEs of the explanatory variables were used in order to make their interpretations intuitively meaningful. Moreover, AMEs instead of the Marginal Effects at the Means (MEMs) were used because the former is deemed to be superior to the latter [30].

All explanatory variables of interest were tested for multicollinearity before fitting them in the regression models and variables with Variance Inflation Factor (VIF) above the 10.0 rule of thumb were dropped. Apart from age, all the remaining explanatory variables were treated as dummy variables because of their categorical nature. It must however be stressed that, North East and Savannah regions had one respondent each and Ahafo region had eight respondents. The few number of respondents led to multicollinearity; hence, the variable "region" was recoded to merge "North East" and "Savannah" regions to "Northern" region. Similarly, "Ahafo", "Bono" and "Bono East" were merged and recorded as "Brong-Ahafo" region for same reasons. Doing so is justified because these regions were each one administrative region before the demarcation of Ghana's new regions in 2019.

\section{Findings \\ Background information of respondents}

Total of 1605 responses were received from the webbased survey. After data cleaning 1159 complete responses were retained representing $72 \%$ return rate. Approximately $62 \%$ of the respondents were females; the mean age was $31.91 \pm 10$. Majority of the respondents were married (54\%) and Christian (92\%); likewise, respondents from Greater Accra region (22\%) dominated while respondents with at least diploma qualification constituted approximately 35\%; $77 \%$ of the respondents worked in government-owned facilities with $34 \%$ of them working for 2-5 years; out of the 1095 respondents who answered the question on whether they worked in a COVID-19 treatment centre at the time of answering the questionnaire, $74 \%$ of them said no. Overall, over $90 \%$ of the 1126 respondents were clinical frontline staff and in terms of the professional category of the respondents, 
Table 1 Background characteristics of respondents

\begin{tabular}{|c|c|c|}
\hline \multirow{2}{*}{$\begin{array}{l}\text { Variables } \\
\text { Sex of respondent }\end{array}$} & \multicolumn{2}{|c|}{ Statistics } \\
\hline & Freq & Percent \\
\hline Male & 436 & 38.18 \\
\hline Female & 706 & 61.82 \\
\hline Total & 1142 & 100.00 \\
\hline \multicolumn{3}{|l|}{ Age range of respondent } \\
\hline $18-23$ & 50 & 4.66 \\
\hline $24-29$ & 302 & 28.15 \\
\hline $30-35$ & 503 & 46.88 \\
\hline $36-41$ & 168 & 15.66 \\
\hline $42-47$ & 31 & 2.89 \\
\hline $48+$ & 19 & 1.77 \\
\hline Total & 1073 & 100.00 \\
\hline \multicolumn{3}{|l|}{ Marital status of respondent } \\
\hline Divorced/widowed & 17 & 1.54 \\
\hline Living together & 19 & 1.72 \\
\hline Married & 594 & 53.85 \\
\hline Never married & 457 & 41.43 \\
\hline Separated & 16 & 1.45 \\
\hline Total & 1103 & 100.00 \\
\hline \multicolumn{3}{|l|}{ Religious affiliation of respondent } \\
\hline Christian & 1016 & 92.03 \\
\hline Moslem/Traditionalist/other & 88 & 7.97 \\
\hline Total & 1104 & 100.00 \\
\hline \multicolumn{3}{|c|}{ Region of residence of respondent } \\
\hline Ashanti & 61 & 5.77 \\
\hline Ahafo & 9 & 0.85 \\
\hline Brong Ahafo & 38 & 3.60 \\
\hline Bono East & 45 & 4.26 \\
\hline Central & 85 & 8.04 \\
\hline Eastern & 98 & 9.27 \\
\hline Greater Accra & 228 & 21.57 \\
\hline Northern/North East & 63 & 5.96 \\
\hline Savannah & 13 & 1.23 \\
\hline Upper East & 60 & 5.68 \\
\hline Upper West & 33 & 3.12 \\
\hline V olta/Oti & 219 & 20.72 \\
\hline Western/Western North & 105 & 9.93 \\
\hline Total & 1057 & 100.00 \\
\hline \multicolumn{3}{|l|}{ Educational level of respondent } \\
\hline Certificate & 349 & 31.08 \\
\hline Diploma & 391 & 34.82 \\
\hline Bachelors & 335 & 29.83 \\
\hline Masters/PhD/fellowship & 48 & 4.27 \\
\hline Total & 1123 & 100.00 \\
\hline Student & 52 & 4.62 \\
\hline Allied health & 186 & 16.52 \\
\hline Medical officer & 20 & 1.78 \\
\hline Midwife & 197 & 17.50 \\
\hline Nurse & 560 & 49.73 \\
\hline
\end{tabular}

Table 1 (continued)

\begin{tabular}{|c|c|c|}
\hline \multirow{2}{*}{$\frac{\text { Variables }}{\text { Pharmacist }}$} & \multicolumn{2}{|c|}{ Statistics } \\
\hline & 19 & 1.69 \\
\hline Other health staff & 92 & 8.17 \\
\hline Total & 1126 & 100.00 \\
\hline \multicolumn{3}{|c|}{ Workplace of respondent } \\
\hline Government & 829 & 77.40 \\
\hline Private & 71 & 6.63 \\
\hline Mission & 113 & 10.55 \\
\hline Quasi/Other & 58 & 5.42 \\
\hline Total & 1071 & 100.00 \\
\hline \multicolumn{3}{|c|}{ Work experience of health staff } \\
\hline 1 year or less & 238 & 22.60 \\
\hline $2-5$ years & 358 & 34.00 \\
\hline $6-9$ years & 289 & 27.45 \\
\hline 10 years or more & 168 & 15.95 \\
\hline Total & 1053 & 100.00 \\
\hline \multicolumn{3}{|c|}{ Respondents working in COVID-19 treatment centre } \\
\hline No & 813 & 74.25 \\
\hline Yes & 282 & 25.75 \\
\hline Total & 1095 & 100.00 \\
\hline \multicolumn{3}{|c|}{ Participate in COVID-19 vaccine trial } \\
\hline No & 393 & 52.33 \\
\hline Yes & 358 & 47.67 \\
\hline Total & 751 & 100.00 \\
\hline \multicolumn{3}{|c|}{ Advice someone to participate in a COVID-19 vaccine trial } \\
\hline No & 354 & 47.64 \\
\hline Yes & 389 & 52.36 \\
\hline Total & 743 & 100.00 \\
\hline \multicolumn{3}{|c|}{ Accept to be immunized with a COVID-19 vaccine } \\
\hline No & 223 & 29.85 \\
\hline Yes & 524 & 70.15 \\
\hline Total & 747 & 100.00 \\
\hline \multicolumn{3}{|c|}{ Advice someone to be immunized with a COVID-19 vaccine } \\
\hline No & 184 & 24.76 \\
\hline Yes & 559 & 75.24 \\
\hline Total & 743 & 100.00 \\
\hline
\end{tabular}

Bold values indicate occupation/professional category

Source: field Data (2020); Legend: COVID-19 (Coronavirus Disease 2019)

nurses dominated representing nearly $50 \%$ followed by midwives (18\%). The least category was medical officers who constituted barely $2 \%$ of the study sample (see Table 1).

\section{Willingness to participate in COVID-19 vaccine trial and uptake the vaccine}

It was observed that $48 \%$ of the respondents said they will participate in a COVID-19 vaccine trial when given the opportunity; $52 \%$ will advise someone to participate in the COVID-19 vaccine trial; $70 \%$ of the respondents 
Table 2 Cross-tabulations on willingness to participate in COVID-19 vaccine trial and uptake the vaccine

\begin{tabular}{|c|c|c|c|c|c|c|}
\hline \multirow{2}{*}{$\begin{array}{l}\text { Variables } \\
\text { Sex }\end{array}$} & \multicolumn{3}{|c|}{ Accept COVID-19 vaccination } & \multicolumn{3}{|c|}{ Participate in COVID-19 vaccine trial } \\
\hline & No & Yes & Total & No & Yes & Total \\
\hline Male & 75 & 232 & 307 & 144 & 165 & 309 \\
\hline Female & 146 & 288 & 434 & 246 & 190 & 436 \\
\hline Total & 221 & 520 & 741 & 390 & 355 & 745 \\
\hline \multicolumn{7}{|l|}{ Age range } \\
\hline $18-23$ & 7 & 26 & 33 & 23 & 11 & 34 \\
\hline $24-29$ & 54 & 140 & 194 & 106 & 89 & 195 \\
\hline $30-35$ & 95 & 234 & 329 & 165 & 164 & 329 \\
\hline $36-41$ & 35 & 74 & 109 & 49 & 59 & 108 \\
\hline $42-47$ & 9 & 13 & 22 & 15 & 8 & 23 \\
\hline 48 and above & 5 & 6 & 11 & 8 & 4 & 12 \\
\hline Total & 205 & 493 & 698 & 366 & 335 & 701 \\
\hline \multicolumn{7}{|l|}{ Marital status } \\
\hline Divorced & 3 & 4 & 7 & 4 & 3 & 7 \\
\hline Living together & 6 & 10 & 16 & 11 & 5 & 16 \\
\hline Married & 114 & 254 & 368 & 159 & 205 & 364 \\
\hline Never married & 90 & 231 & 321 & 162 & 159 & 321 \\
\hline Separated & 3 & 6 & 9 & 6 & 3 & 9 \\
\hline Widowed & 0 & 2 & 2 & 1 & 1 & 2 \\
\hline Total & 216 & 507 & 723 & 343 & 376 & 719 \\
\hline \multicolumn{7}{|l|}{ Educational level } \\
\hline Certificate & 63 & 154 & 217 & 107 & 112 & 219 \\
\hline Diploma & 76 & 174 & 250 & 129 & 122 & 251 \\
\hline Bachelor degree & 76 & 155 & 231 & 131 & 101 & 232 \\
\hline Master degree & 6 & 21 & 27 & 13 & 14 & 27 \\
\hline Fellowship & 0 & 2 & 2 & 1 & 1 & 2 \\
\hline $\mathrm{PhD}$ & 0 & 3 & 3 & 0 & 3 & 3 \\
\hline Total & 221 & 509 & 730 & 381 & 353 & 734 \\
\hline \multicolumn{7}{|l|}{ Professional category } \\
\hline Allied health professional & 29 & 84 & 113 & 45 & 67 & 112 \\
\hline Medical officer & 3 & 14 & 17 & 6 & 11 & 17 \\
\hline Midwife & 43 & 76 & 119 & 63 & 56 & 119 \\
\hline Nurse & 116 & 260 & 376 & 175 & 196 & 371 \\
\hline Pharmacist & 4 & 7 & 11 & 4 & 7 & 11 \\
\hline Student & 6 & 28 & 34 & 17 & 17 & 34 \\
\hline Other & 19 & 46 & 65 & 37 & 30 & 67 \\
\hline Total & 220 & 515 & 735 & 347 & 384 & 731 \\
\hline \multicolumn{7}{|l|}{ Facility ownership } \\
\hline Public/government & 156 & 382 & 538 & 277 & 262 & 539 \\
\hline Private & 25 & 30 & 55 & 32 & 24 & 56 \\
\hline Mission & 16 & 52 & 68 & 26 & 42 & 68 \\
\hline Quasi-government & 14 & 13 & 27 & 19 & 8 & 27 \\
\hline Other & 6 & 5 & 11 & 10 & 1 & 11 \\
\hline Total & 217 & 482 & 699 & 364 & 337 & 701 \\
\hline \multicolumn{7}{|l|}{ Health facility level } \\
\hline Teaching hospital & 23 & 44 & 67 & 42 & 26 & 68 \\
\hline Regional hospital & 9 & 18 & 27 & 15 & 12 & 27 \\
\hline District hospital & 83 & 176 & 259 & 152 & 107 & 259 \\
\hline Polyclinic/clinic & 30 & 53 & 83 & 43 & 40 & 83 \\
\hline
\end{tabular}


Table 2 (continued)

\begin{tabular}{|c|c|c|c|c|c|c|}
\hline \multirow{2}{*}{$\begin{array}{l}\text { Variables } \\
\text { Sex }\end{array}$} & \multicolumn{3}{|c|}{ Accept COVID-19 vaccination } & \multicolumn{3}{|c|}{ Participate in COVID-19 vaccine trial } \\
\hline & No & Yes & Total & No & Yes & Total \\
\hline Health centre & 47 & 117 & 164 & 67 & 98 & 165 \\
\hline CHPS compound & 21 & 64 & 85 & 38 & 47 & 85 \\
\hline Total & 213 & 472 & 685 & 357 & 330 & 687 \\
\hline \multicolumn{7}{|l|}{ Work in COVID-19 centre } \\
\hline No & 161 & 360 & 521 & 265 & 257 & 522 \\
\hline Yes & 56 & 134 & 190 & 105 & 86 & 191 \\
\hline Total & 217 & 494 & 711 & 370 & 343 & 713 \\
\hline \multicolumn{7}{|c|}{ Voluntarily test for COVID-19 } \\
\hline \multicolumn{7}{|l|}{ Very unlikely } \\
\hline Unlikely & 10 & 26 & 36 & 23 & 13 & 36 \\
\hline Undecided & 22 & 28 & 50 & 34 & 17 & 51 \\
\hline Likely & 26 & 39 & 65 & 44 & 23 & 67 \\
\hline Very likely & 85 & 223 & 308 & 154 & 155 & 309 \\
\hline Total & 79 & 206 & 285 & 137 & 148 & 285 \\
\hline \multicolumn{7}{|l|}{ Ever tested for COVID-19 } \\
\hline Yes & 76 & 171 & 247 & 121 & 127 & 248 \\
\hline No & 140 & 342 & 482 & 262 & 223 & 485 \\
\hline Prefer not to disclose & 5 & 4 & 9 & 6 & 3 & 9 \\
\hline Total & 221 & 517 & 738 & 389 & 353 & 742 \\
\hline
\end{tabular}

Source: field Data (2020); Legend: CHPS (Community-based health planning and services)

indicated their willingness to accept the COVID-19 vaccine when given the opportunity, and $75 \%$ will advise someone to take the vaccine (see Table 1). Table 2 shows details of disaggregated responses on willingness to participate in a vaccine trial and accept the vaccine according to age, gender, religion, marital status, educational qualification, type of health facility and professional category.

\section{Determinants of voluntary participation in COVID-19 vaccine trial}

Significant determinants of likelihood of participating in COVID-19 vaccine trial were: age, religious affiliation and ownership of health facility where the staff works. For instance, health workers aged 36-41 years were 28 times more likely to voluntarily participate in a COVID19 vaccine trial $(\mathrm{AME}=0.28, \mathrm{SE}=0.16, p<0.1)$ than persons aged 18-23 years; persons aged 48 years or more were 38 times less likely to advise someone to participate in a COVID-19 vaccine trial $(\mathrm{AME}=-38, \mathrm{SE}=0.20$, $p<0.1)$ relative to persons aged $18-23$ years. Non-Christians were 21 more likely to voluntarily participate in a COVID-19 vaccine trial $(\mathrm{AME}=21, \mathrm{SE}=0.09, p<0.05)$ and recommend same to someone $(\mathrm{AME}=19, \mathrm{SE}=0.09$, $p<0.05)$ relative to Christians. Relative to health staff working in government-owned health facilities, those working in Mission health facilities were more likely to voluntarily participate in COVID-19 vaccine trial $(\mathrm{AME}=18, \mathrm{SE}=0.07, p<0.05)$ and recommend same to others $(\mathrm{AME}=15, \mathrm{SE}=0.07, p<0.05)$. Conversely, staff working in quasi-government facilities were 27 times less likely to participate in COVID-19 vaccine trial $(\mathrm{AME}=-27, \mathrm{SE}=0.08, p<0.01)$ and recommend same to others $(\mathrm{AME}=-24, \mathrm{SE}=0.09, p<0.01)$ compared to those in the government-owned facilities (see Table 3).

\section{Correlates of staff willingness to uptake COVID-19 vaccine}

The results further revealed that females were 11 times less likely to accept a COVID-19 vaccine when given the opportunity compared to males $(\mathrm{AME}=-11, \mathrm{SE}=0.04$, $p<0.05)$; females were also 10 times less likely to advise someone to take the vaccine $(\mathrm{AME}=-10, \mathrm{SE}=0.04$, $p<0.05)$ than their male counterparts. Older respondents (48 years and above) were 35 times less likely to recommend a COVID-19 vaccine to someone (AME $=-35$, $\mathrm{SE}=0.21, \quad p<0.1)$ relative to younger age group (18-23 years).

Similarly, persons whose marital status was "living together" were 33 times less likely to recommend a COVID-19 vaccine to someone relative to divorced/ widowed respondents $(\mathrm{AME}=-33, \mathrm{SE}=0.20, p<0.1)$. Non-Christians were 20 times more likely to accept a COVID-19 vaccine $(\mathrm{AME}=0.20, \mathrm{SE}=0.06, p<0.01)$ and 20 times more likely to recommend same to others 
Table 3 Probit regression estimates of determinants of willingness to participate in COVID-19 vaccine trial

\begin{tabular}{|c|c|c|}
\hline & Self & Someone \\
\hline \multicolumn{3}{|l|}{ Sex (Ref: male) } \\
\hline Female & $-0.04(0.05)$ & $-0.05(0.05)$ \\
\hline \multicolumn{3}{|l|}{ Age (Ref: 18-23) } \\
\hline $24-29$ & $0.22(0.15)$ & $-0.01(0.14)$ \\
\hline $30-35$ & $0.16(0.15)$ & $-0.05(0.15)$ \\
\hline $36-41$ & $0.28^{*}(0.16)$ & $0.04(0.16)$ \\
\hline $42-47$ & $0.07(0.19)$ & $-0.19(0.19)$ \\
\hline $48+$ & $-0.11(0.20)$ & $-0.38^{*}(0.20)$ \\
\hline \multicolumn{3}{|c|}{ Marital status (Ref: divorced/widowed) } \\
\hline Living together & $-0.24(0.23)$ & $-0.30(0.23)$ \\
\hline Married & $0.06(0.19)$ & $0.06(0.20)$ \\
\hline Never married & $-0.002(0.20)$ & $0.01(0.20)$ \\
\hline Separated & $-0.13(0.28)$ & $-0.17(0.28)$ \\
\hline \multicolumn{3}{|l|}{ Region (Ref: Ashanti) } \\
\hline Ahafo & $0.03(0.26)$ & $-0.00(0.26)$ \\
\hline Brong Ahafo & $0.11(0.15)$ & $0.18(0.15)$ \\
\hline Bono East & $0.08(0.15)$ & $0.09(0.15)$ \\
\hline Central & $-0.03(0.11)$ & $0.01(0.11)$ \\
\hline Eastern & $-0.01(0.11)$ & $0.02(0.11)$ \\
\hline Greater Accra & $0.02(0.10)$ & $-0.01(0.10)$ \\
\hline Northern/North East & $0.05(0.12)$ & $0.01(0.12)$ \\
\hline Savannah & $-0.18(0.17)$ & $0.06(0.21)$ \\
\hline Upper East & $0.18(0.12)$ & $0.13(0.13)$ \\
\hline Upper West & $-0.00(0.15)$ & $0.03(0.15)$ \\
\hline Volta/Oti & $-0.02(0.10)$ & $0.03(0.10)$ \\
\hline Western/Western North & $0.08(0.11)$ & $0.06(0.11)$ \\
\hline \multicolumn{3}{|l|}{ Religion (Christian) } \\
\hline Moslem/traditionalist/other & $0.21^{* *}(0.09)$ & $0.19^{* *}(0.09)$ \\
\hline \multicolumn{3}{|l|}{ Education (Ref: certificate) } \\
\hline Diploma & $0.00(0.06)$ & $0.02(0.06)$ \\
\hline Bachelors & $-0.08(0.06)$ & $-0.04(0.06)$ \\
\hline Masters/PhD/fellowship & $0.06(0.12)$ & $0.06(0.12)$ \\
\hline \multicolumn{3}{|c|}{ Professional category (Ref: student) } \\
\hline Allied health & $0.15(0.10)$ & $0.03(0.10)$ \\
\hline Medical officer & $0.11(0.15)$ & $0.04(0.15)$ \\
\hline Midwife & $-0.02(0.10)$ & $-0.09(0.10)$ \\
\hline Nurse & $0.10(0.09)$ & $-0.02(0.09)$ \\
\hline Pharmacist & $-0.09(0.17)$ & $0.01(0.19)$ \\
\hline Physician assistant & $0.04(0.18)$ & $0.05(0.18)$ \\
\hline \multicolumn{3}{|l|}{ Workplace (Ref: public) } \\
\hline Private & $0.03(0.08)$ & $-0.03(0.08)$ \\
\hline Mission & $0.18^{* * * *}(0.07)$ & $0.15^{* *}(0.07)$ \\
\hline Quasi/Other & $-0.27^{* * *}(0.08)$ & $-0.24^{* * *}(0.09)$ \\
\hline \multicolumn{3}{|l|}{ Experience (1 year or less) } \\
\hline $2-5$ years & $0.05(0.06)$ & $0.05(0.06)$ \\
\hline $6-9$ years & $0.06(0.07)$ & $0.04(0.07)$ \\
\hline 10 years or more & $0.12(0.08)$ & $0.12(0.09)$ \\
\hline \multicolumn{3}{|c|}{ COVID-19 treatment centre (Ref: no) } \\
\hline Yes & $-0.06(0.05)$ & $-0.07(0.05)$ \\
\hline Observations & 569 & 563 \\
\hline
\end{tabular}

Table 3 (continued) Average Marginal Effects; standard errors in parentheses; ${ }^{*} p<0.1,{ }^{* *} p<0.05,{ }^{* * *}$ $p<0.01$

$(\mathrm{AME}=0.17, \mathrm{SE}=0.06, p<0.01)$ than Christians. Persons who had higher education (i.e., Masters/PhD/fellowship qualification) were 16 times more likely to accept a COVID-19 vaccine $(\mathrm{AME}=0.16, \mathrm{SE}=0.08, p<0.1)$ and 19 times more likely recommend same to others (AME $=0.19, \mathrm{SE}=0.07, p<0.01$ ) as compared to those with lower qualification. Staff who worked in private health facilities were 17 times less likely to participate in a COVID-19 vaccine trial $(\mathrm{AME}=-0.17, \mathrm{SE}=0.08$, $p<0.05)$ and 12 times less likely to recommend same to others $(\mathrm{AME}=-0.12, \mathrm{SE}=0.07, p<0.1)$ relative to those working in government-owned facilities. Finally, respondents who worked in quasi-government facilities were 34 less likely to accept a COVID-19 vaccine $(\mathrm{AME}=-34, \mathrm{SE}=0.09, p<0.01)$ and 31 times less likely to recommend such a vaccine to others $(\mathrm{AME}=-31$, $\mathrm{SE}=0.10, p<0.01)$ as compared to staff working in government-owned facilities (see Table 4).

Follow-up questions revealed that the predominant reasons cited by $\mathrm{HCWs}$ for their unwillingness to participate in a COVID-19 vaccine trial and uptake the vaccine included fear, health and safety concerns, mistrust, uncertainty, spiritual and religious beliefs (see Additional file 1 for details).

\section{Discussion}

HCWs are a critical piece of every health system across the globe [31]. Outbreak of the COVID-19 pandemic and the devastating effect on healthcare delivery has reemphasized the important role of HCWs in every health system [32]. Safety of HCWs is particularly central in the response of nations against the pandemic. While some countries have performed relatively better in terms of protecting these frontline critical staff, same cannot be said of the resource-poor countries in the global south like Ghana [7, 33, 34].

There are varied reports on the number of COVID-19 infections and deaths among frontline healthcare workers (HCW) [33]. For instance, WHO African Regional Office estimates over 10000 healthcare workers in Africa were infected with the virus in 2020 [7]. Likewise, the WHO Pan American Regional Office (PAHO) reported that $570000 \mathrm{HCWs}$ were infected and 2500 died due to COVID-19 as at September, 2020 [34]. Even though there is paucity of empirical data on COVID-19 related deaths of HCWs in Ghana, available anecdotal reports suggest over $2000 \mathrm{HCWs}$ have been infected and at least 6 deaths recorded [35]. Even though this study started months before deployment of the COVID-19 vaccine, the current 
Table 4 Probit regression estimates of determinants of willingness to uptake COVID-19 vaccine

\begin{tabular}{|c|c|c|}
\hline & Self & Someone \\
\hline \multicolumn{3}{|l|}{ Sex (Ref: male) } \\
\hline Female & $-0.11^{* *}(0.04)$ & $-0.10^{* *}(0.04)$ \\
\hline \multicolumn{3}{|l|}{ Age (Ref: 18-23) } \\
\hline $24-29$ & $0.03(0.12)$ & $0.02(0.11)$ \\
\hline $30-35$ & $-0.02(0.12)$ & $-0.00(0.12)$ \\
\hline $36-41$ & $-0.07(0.14)$ & $0.03(0.13)$ \\
\hline $42-47$ & $-0.12(0.18)$ & $-0.15(0.18)$ \\
\hline $48+$ & $-0.30(0.21)$ & $-0.35^{*}(0.21)$ \\
\hline \multicolumn{3}{|c|}{ Marital status (ref: divorced/widowed) } \\
\hline Living together & $-0.29(0.21)$ & $-0.33^{*}(0.20)$ \\
\hline Married & $-0.10(0.14)$ & $-0.04(0.13)$ \\
\hline Never married & $-0.11(0.15)$ & $-0.10(0.14)$ \\
\hline Separated & $-0.08(0.22)$ & $-0.25(0.23)$ \\
\hline \multicolumn{3}{|l|}{ Region (Ref: Ashanti) } \\
\hline Ahafo & $0.06(0.24)$ & $0.08(0.22)$ \\
\hline Brong Ahafo & $0.04(0.15)$ & $0.19(0.13)$ \\
\hline Bono East & $-0.08(0.15)$ & $0.07(0.14)$ \\
\hline Central & $0.02(0.10)$ & $0.09(0.10)$ \\
\hline Eastern & $0.11(0.10)$ & $0.15(0.10)$ \\
\hline Greater Accra & $0.05(0.09)$ & $0.09(0.09)$ \\
\hline Northern/North East & $0.02(0.12)$ & $0.07(0.11)$ \\
\hline Savannah & $-0.06(0.24)$ & $-0.04(0.24)$ \\
\hline Upper East & $0.08(0.12)$ & $0.12(0.12)$ \\
\hline Upper West & $-0.01(0.15)$ & $0.16(0.13)$ \\
\hline Volta/Oti & $0.00(0.09)$ & $0.09(0.09)$ \\
\hline Western/Western North & $0.13(0.10)$ & $0.12(0.10)$ \\
\hline \multicolumn{3}{|l|}{ Religion (Ref: Christian) } \\
\hline Moslem/Traditionalist/other & $0.20^{* * * *}(0.06)$ & $0.17^{* * *}(0.06)$ \\
\hline \multicolumn{3}{|l|}{ Education (Ref: certificate) } \\
\hline Diploma & $-0.01(0.05)$ & $0.03(0.05)$ \\
\hline Bachelors & $-0.01(0.05)$ & $0.03(0.05)$ \\
\hline Masters/PhD/fellowship & $0.16^{*}(0.08)$ & $0.19^{* * *}(0.07)$ \\
\hline \multicolumn{3}{|c|}{ Professional category (Ref: student) } \\
\hline Allied health & $-0.00(0.09)$ & $0.05(0.09)$ \\
\hline Medical officer & $0.07(0.13)$ & $0.09(0.13)$ \\
\hline Midwife & $0.01(0.09)$ & $0.01(0.09)$ \\
\hline Nurse & $-0.03(0.08)$ & $0.02(0.08)$ \\
\hline Pharmacist & $-0.10(0.18)$ & $0.10(0.14)$ \\
\hline Physician assistant & $0.08(0.14)$ & $0.14(0.12)$ \\
\hline \multicolumn{3}{|l|}{ Workplace (Ref: public) } \\
\hline Private & $-0.17^{* *}(0.08)$ & $-0.12^{*}(0.07)$ \\
\hline Mission & $0.10^{*}(0.06)$ & $0.05(0.05)$ \\
\hline Quasi/other & $-0.34^{* * *}(0.09)$ & $-0.31^{* * * *}(0.10)$ \\
\hline \multicolumn{3}{|l|}{ Experience (Ref: 1 year or less) } \\
\hline $2-5$ years & $-0.07(0.06)$ & $-0.08(0.05)$ \\
\hline $6-9$ years & $-0.02(0.06)$ & $-0.03(0.06)$ \\
\hline 10 years or more & $-0.00(0.08)$ & $-0.02(0.07)$ \\
\hline \multicolumn{3}{|c|}{ COVID-19 treatment centre (Ref: No) } \\
\hline Yes & $-0.01(0.04)$ & $0.01(0.04)$ \\
\hline Observations & 568 & 565 \\
\hline
\end{tabular}

Table 4 (continued)

Average Marginal Effects; standard errors in parentheses; ${ }^{*} p<0.1,{ }^{* *} p<0.05,{ }^{* * *}$ $p<0.01$

literature suggests high mortality rates among HCWs due to COVID-19 is largely due to limited vaccination coverage and inadequate supply of personal protective equipment (PPEs) [33].

It is therefore not out of place that many countries globally have prioritized HCWs for vaccination against COVID-19 [15, 36-39]. Unfortunately, the all-time record breakthrough in development and deployment of the COVID-19 vaccine has induced scepticisms and safety concerns on the vaccine. These concerns have impacted negatively on acceptance of this important intervention to control and eventually eradicate the pandemic.

Ghana's history on resistance to vaccine trials and vaccine uptake is not too different from many African countries. Key among the historical antecedents are the suspension of the Ebola vaccine Phase 1 trial in 2015 [12] and early resistance to the ongoing malaria vaccine trial (RTS,S) which eventually started in April, 2019 [13] after successful stakeholder engagements. Reviewed literature points to the huge mass media misinformation (i.e., linkage with 5G technology and so-called vaccine chip implantation in the human body) on the COVID-19 vaccine as a major barrier to acceptance and uptake. This misinformation is coupled with the unbridled negative influence by some religious leaders and groups on public opinion concerning vaccine trials and vaccine uptake including the COVID-19 vaccine [40].

Unfortunately, there is limited empirical evidence on sources of the skepticism and vaccine trial hesitancy, especially among HCWs. Available literature has largely concentrated on community perceptions and willingness to participate in the COVID-19 trials and subsequently, accept the vaccine. This study is therefore timely, because local pharmaceutical companies have intensified efforts to locally produce some of the COVID-19 vaccines in Ghana [23] and perspectives of HCWs is crucial to a successful rollout of this intervention.

As demonstrated in this study, willingness to participate in a COVID-19 vaccine trial was $48 \%$ compared to $70 \%$ in a similar study by Kitonsa et al. [41] among HCWs in Uganda. Likelihood of accepting the COVID-19 vaccine was however high (70\%) suggesting low vaccine hesitancy among HCWs in Ghana. Feedback from the HCWs suggests Ghana is possibly among the few countries with a relatively high vaccine acceptance rate among HCWs in the sub-region relative to Nigeria [42], DRC [43] and outside the sub-region such as France [18]. 
It was observed that gender, age, religious affiliation and ownership of health facility where the staff works are significant correlates of HCWs' likelihood of participating in a COVID-19 vaccine trial and accept the vaccine. Older staff and those of Christian religious faith were found to be more risk-averse as far as participation in the COVID-19 vaccine trial and uptake of the vaccine are concerned. Additionally, staff working in Mission facilities were more likely to participate in COVID-19 vaccine trial relative to government-owned facilities. This observation corroborates findings of similar studies which intimated personal and institutional level characteristics of HCWs potentially influence likelihood of accepting the COVID-19 vaccine trial and vaccine uptake [44, 45]. Future qualitative studies could help "dig deep" into reasons for these dynamics since the current study was a survey that did not explore the depth in terms of these views.

It was also found that females and older staff were more risk-averse as far as likelihood of taking the COVID-19 vaccine is concerned. Enitan et al. [38] found similar results in the case of Nigeria. Similarly, staff with relatively higher educational qualification were more likely to uptake the COVID-19 compared to those with lower educational qualifications which suggests limited exposure to information potentially entrenches misconceptions on the COVID-19 vaccine and hinders its uptake.

Staff working in private health facilities were less likely to accept the COVID-19 immunization compared to staff working in government-owned facilities. Further research is required to unearth reasons for these differentials in the various categories of health facilities, particularly in terms of type, level and the mix of staff found in these types of facilities to inform tailor made vaccine promotion campaigns among the different cadre of HCWs based on their peculiar needs.

An integrated yet multisectoral approach is imperative to ensure targeted vaccine promotion campaigns to address hesitancy among HCWs and the community at large as alluded to by Afolabi and Ilesanmi [40] and in the case of South Sudan [46]. Agyekum et al. [47] arrived at similar findings on vaccine hesitancy among health workers in Ghana, thus corroborating results of this study and the need for immediate interventions to further improve perceptions of HCWs since they are expected to be good ambassadors of this public health intervention to help control the pandemic.

Finally, concerns of fear, safety, mistrust and uncertainty intimated by the HCWs must be addressed through tailored messaging to promote goodwill and confidence in the vaccine as alluded to by Dror et al. [48]. Addressing concerns of HCWs on COVID-19 vaccine will help allay their fears and empower them as advocates to champion effective rollout of the vaccine to the general population.

\section{Conclusion}

Likelihood of accepting the COVID-19 vaccine was found to be high among HCWs in Ghana albeit willingness to volunteer for the vaccine trial was relatively low. Nonetheless, this observation suggests Ghana, compared to Nigeria [42] and DRC [43], is doing better. A similar study in Uganda [41] revealed a vaccine acceptance rate of over $70 \%$. The current findings on the acceptance rate demonstrates some level of confidence on the COVID-19 vaccine among HCWs in Ghana which needs to be sustained through continuous targeted public health education and sensitization seminars for HCWs. COVID-19 vaccine rollout interventions for the global south should therefore be guided by these country-specific context to promote effective implementation and success of these interventions.

Even though the findings reported in this paper are exploratory and might not entirely be conclusive, the results have adduced baseline empirical evidence on the need for more follow-up mixed methods studies to fully understand experiences and reasons for vaccine acceptance and hesitancy among HCWs. HCWs are an important source of health information and validation for the general public and their perspectives on the COVID-19 vaccine is critical to influencing public opinion on vaccinations in general.

Additionally, continuous engagement with HCWs by health managers and policy experts throughout the value chain on vaccine trials and deployment will promote trust and confidence which will likely trickle down to the general population through advocacy by $\mathrm{HCWs}$ on public health benefits of vaccination.

Finally, there is the need for effective stakeholder engagements towards championing vaccinations uptake as a public health intervention. Government agencies, Civil Society Organizations (CSOs), health professional groups should lead in mobilizing resources for these campaigns. The Ghana Medical Association (GMA), Ghana Registered Nurses and Midwives Association (GRNMA) and allied health professional groups should lead the crusade on educating their members as agents of change. Likewise, these health professional groups should prioritize scientific research to inform health advocacies and public education on the COVID-19 vaccine.

\section{Limitations}

It is important to acknowledge that this survey was conducted several months before the deployment of the COVID-19 vaccine in Ghana. In light of this lag in time, perspectives are likely to be different among the same 
study populations after some HCWs have now taken the vaccines and experienced reactogenicity of the vaccine. Moreover, the survey was web-based and might have exposed the design to selection bias since persons who owned a smartphone might have predominantly self-selected into the study. Nonetheless, use of research assistants to administer the questionnaire in-person to respondents who could not access the web-based survey minimized impact of the potential selection bias. Additionally, the nationwide scope with large sample size coupled with the use of pre-tested and validated tools render the findings valid and compelling.

\section{Implications for public health policy}

While acknowledging the above limitations associated with this study, the following policy recommendations are proposed based on the study findings:

1. Health professionals' regulatory councils in Ghana in collaboration with their employers and Health Facilities Regulatory Agency (HeFRA) should consider instituting mandatory vaccination for $\mathrm{HCWs}$ as a safety precaution. Section 22(1) of the Public Health Act, 2012 (Act 851) could be invoked for this purpose

2. The Ministry of Health $(\mathrm{MoH})$ and Food and Drugs Authority (FDA) of Ghana should institute mandatory community-based stakeholder engagement protocols throughout the value chain of vaccine development, trials and rollout to enhance trust and acceptance in vaccines.

3. Health promotion unit of the Ghana Health Service (GHS) in collaboration with the information services department should collaborate to develop targeted national health information policy guidelines on vaccines to address the peculiar knowledge gaps of $\mathrm{HCWs}$ and the general population on vaccination.

4. Ministry of Health $(\mathrm{MoH})$ and its implementing agents should collaborate with religious institutions and their leadership to identify and empower community champions to advocate for vaccine uptake.

\footnotetext{
Abbreviations

AME: Average Marginal Effects; AU: African Union; CDC: Centres for Disease Control and Prevention; COVID-19: Coronavirus Disease 2019; CSOs: Civil Society Organizations; DRC: Democratic Republic of Congo; EUA: Emergency use authorization; GHS: Ghana Health Service; GMA: Medical Association of Ghana; GRNMA: Ghana Registered Nurses and Midwives Association; GSK: GlaxoSmithKline; HCWs: Health Care Workers; MEMs: Marginal Effects at the Means; MoH: Ministry of Health; NPIs: Non-pharmaceutical interventions; PAHO: Pan American Regional Office; REC: Research Ethics Committee; SE: Standard error; UHAS: University of Health and Allied Sciences; VIF: Variance Inflation Factor; WHO: World Health Organization.
}

\section{Supplementary Information}

The online version contains supplementary material available at https://doi. org/10.1186/s12960-021-00657-1.

Additional file 1: Table S1. Verbatim reasons cited for unwillingness to participate in a COVID-19 vaccine Trial and uptake the vaccine.

\section{Acknowledgements}

The researchers appreciate and acknowledge the support of the community health nurses who were involved in the data collection and all respondents who voluntarily participated in the study. Also, online databases of the Ghana Health Service (GHS), African Union (AU) Centres for Disease Control and Prevention (CDC) and the World Health Organization (WHO) were used for the literature review and hereby acknowledged. Service of Mustapha Immurana in the analysis of the data for this study is hereby acknowledged.

\section{Authors' contributions}

RKA, MG: provided conceptualization direction, RKA: analysis, RKA, MG, EKA, SO: review and supervision, RKA collected data, RKA: wrote initial draft. All authors read and approved the final manuscript.

Funding

No funding was provided for this study.

\section{Availability of data and materials}

All data generated or analyzed during this study are included in this published article and its Additional file 1.

\section{Declarations}

Ethical approval and consent to participate

The study was approved by the Research Ethics Committee (REC) of the University of Health and Allied Sciences, Ghana (Clearance Number: UHAS-REC A.1 [6]20-21). Informed consent was obtained from all subjects via the webbased questionnaire before they were given access to the questions, Thus, participants who ticked "No" or "Disagree" integrated within the online tool were automatically denied access to the questions.

\section{Consent for publication}

All authors of this manuscript have consented to publish this work.

\section{Competing interests}

The authors declare there is no conflict of interest.

Received: 30 June 2021 Accepted: 10 September 2021

Published online: 06 November 2021

\section{References}

1. World Health Organization (WHO). Official Website. WHO Coronavirus (COVID-19) Dashboard; 2021. https://covid19.who.int/. Accessed 24 March 2021.

2. Centres for Disease Control and Prevention (CDC). Coronavirus Disease 2019 (COVID-19): Latest updates on the COVID-19 crisis from Africa CDC; 2021. https://africacdc.org/covid-19/. Accessed 24 June 2021.

3. Ghana Health Service (GHS). Official website. https://www.ghanahealt hservice.org/covid19/). Accessed 1 March 2021.

4. World Health Organization (WHO) Official Website. Coronavirus 2019 Vaccines; 2021. https://www.who.int/emergencies/diseases/novel-coron avirus-2019/covid-19-vaccines. Accessed 14 March 2021.

5. Bandyopadhyay S, Baticulon RE, Kadhum M, Alser M, Ojuka DK, Badereddin Y, Khundkar R. Infection and mortality of healthcare workers worldwide from COVID-19: a systematic review. BMJ Global Health. 2020;5(12):e003097.

6. World Health Organization (WHO). Health workers: a global profile. Geneva; 2020. https://www.who.int/whr/2006/06_chap1_en.pdf. Accessed 23 June 2021 
7. African Regional Office (AFRO)/World Health Organization (WHO/OMS) Over 10,000 Health Workers in Africa Infected with COVID-19. 2020 (cited 2020 Jul 23); 2020. https://www.afro.who.int/news/over-10-000-healt hworkers-africa-infected-covid-19.

8. Tenforde MW. Effectiveness of Pfizer-BioNTech and moderna vaccines against COVID-19 among hospitalized adults aged $\geq 65$ years—United States, January-March 2021. MMWR. Morbidity and Mortality Weekly Report, 70. 2021.

9. Baden LR, El Sahly HM, Essink B, Kotloff K, Frey S, Novak R, Zaks T. Efficacy and safety of the mRNA-1273 SARS-CoV-2 vaccine. N Engl I Med. 2021;384(5):403-16.

10. Polack FP, Thomas SJ, Kitchin N, Absalon J, Gurtman A, Lockhart S, Gruber WC. Safety and efficacy of the BNT162b2 mRNA Covid-19 vaccine. N Engl J Med. 2020;383(27):2603-15.

11. Sadoff J, Gray G, Vandebosch A, Cárdenas V, Shukarev G, Grinsztejn B, Douoguih M. Safety and efficacy of single-dose Ad26 COV2 S vaccine against COVID-19. N Engl J Med. 2021;384:2187-201.

12. Kummervold PE, Schulz WS, Smout E, Fernandez-Luque L, Larson HJ. Controversial Ebola vaccine trials in Ghana: a thematic analysis of critiques and rebuttals in digital news. BMC Public Health. 2017;17(1):1-24.

13. Petryna A. Ethical variability: drug development and globalizing clinical trials. Am Ethnol. 2005. https://doi.org/10.1525/ae.2005.32.2.183.

14. World Health Organization (WHO), Ghana. Malaria vaccine pilot launched in Ghana: Health officials and community leaders gather to celebrate the landmark launch, 02 May 2019. https://www.afro.who.int/news/malariavaccine-pilot-launched-ghana. Accessed 10 Aug 2021.

15. Gagneux-Brunon A, Detoc M, Bruel S, Tardy B, Rozaire O, Frappe P, Botelho-Nevers E. Intention to get vaccinations against COVID-19 in French healthcare workers during the first pandemic wave: a cross-sectional survey. J Hosp Infect. 2021;108:168-73. https://doi.org/10.1016/j. jhin.2020.11.020 (Epub 2020 Nov 28).

16. Nzaji KM, Ngombe KL, Mwamba NG, Ndala BDB, Miema MJ, Lungoyo LC, Mwimba LB, Bene CMA, Musenga ME. Acceptability of vaccination against COVID-19 among healthcare workers in the Democratic Republic of the Congo. Pragmat Obs Res. 2020;11:103-9. https://doi.org/10.2147/ POR.S271096.

17. Danchin M, Biezen R, Manski-Nankervis JA, Kaufman J, Leask J. Preparing the public for COVID-19 vaccines: How can general practitioners build vaccine confidence and optimise uptake for themselves and their patients? Aust J Gen Pract. 2020;49(10):625-9. https://doi.org/10.31128/ AJGP-08-20-5559.

18. Verger P, Scronias D, Dauby N, Adedzi KA, Gobert C, Bergeat M, Gagneur A, Dubé E. Attitudes of healthcare workers towards COVID-19 vaccination: a survey in France and French-speaking parts of Belgium and Canada, 2020. Euro Surveill. 2021;26(3):2002047. https://doi.org/10.2807/ 1560-7917.ES.2021.26.3.2002047.

19. Netea MG, van der Meer JW, van Crevel R. BCG vaccination in health care providers and the protection against COVID-19. J Clin Invest. 2021;131(2): e145545. https://doi.org/10.1172/JCl145545.

20. Unroe KT, Evans R, Weaver L, Rusyniak D, Blackburn J. Willingness of longterm care staff to receive a COVID-19 vaccine: a single state survey. J Am Geriatr Soc. 2021;69(3):593-9. https://doi.org/10.1111/jgs.17022 (Epub 2021 Jan 13)

21. Shekhar R, Sheikh AB, Upadhyay S, Singh M, Kottewar S, Mir H, Barrett E, Pal S. COVID-19 vaccine acceptance among health care workers in the United States. Vaccines (Basel). 2021;9(2):119. https://doi.org/10.3390/ vaccines 9020119

22. Qattan AMN, Alshareef N, Alsharqi O, Al Rahahleh N, Chirwa GC, AlHanawi MK. Acceptability of a COVID-19 vaccine among healthcare workers in the Kingdom of Saudi Arabia. Front Med (Lausanne). 2021;8: 644300. https://doi.org/10.3389/fmed.2021.644300.

23. Bloomberg. Prognosis: Ghana drugmakers approach AstraZeneca on vaccine production. Updated on April 26, 2021, 2:01 PM GMT; 2021. https:// www.bloomberg.com/news/articles/2021-04-26/ghana-drugmakersapproach-strazeneca-about-local-vaccine-making. Accessed 23 June 2021

24. World Health Organization (WHO) Official Website (2021). COVID-19 vaccine doses shipped by the COVAX Facility head to Ghana, marking beginning of global rollout. https://www.who.int/news/item/24-02-2021covid-19-vaccine-doses-shipped-by-the-covax-facility-head-to-ghanamarking-beginning-of-global-rollout. Accessed 14 Mar 2021.
25. Ghana Health Service (GHS). Health Sector Human Resources Annual Report. Ministry of Health, Accra; 2018.

26. Asamani JA, Amertil NP, Ismaila H, Francis AA, Chebere MM, NabyongaOrem J. Nurses and midwives demographic shift in Ghana-the policy implications of a looming crisis. Hum Resour Health. 2019;17(1):1-5.

27. Krejcie RV, Morgan DW. Determining sample size for research activities. Educ Psychol Measur. 1970;30(3):607-10

28. Cameron AC, Trivedi PK. Microeconometrics: methods and applications. New York: Cambridge University Press; 2005.

29. Cameron AC, Trivedi PK. Microeconometrics using stata. Rev. College Station: Stata Press; 2010

30. Williams R. Using the margins command to estimate and interpret adjusted predictions and marginal effects. Stand Genomic Sci. 2012;12(2):308-31.

31. World Health Organization (WHO). Global strategy on human resources for health: workforce 2030. ISBN 978924151113 1, Geneva; 2016.

32. World Health Organization (WO). Pulse survey on continuity of essential health services during the COVID-19 pandemic: Interim Report, 27th August, 2020. Geneva; 2020.

33. Erdem $H$, Lucey DR. Healthcare worker infections and deaths due to COVID-19: a survey from 37 nations and a call for WHO to post national data on their website. Int J Infect Dis. 2021;102:239.

34. PanAmerican Health Organization (PAHO)/World Health Organization (WHO). COVID-19 has Infected Some 570.000 Health Workers and Killed 2,500 in the Americas. 2020 (cited 2020 Sep 27). https://www.paho.org/ en/news/2-9-2020-covid-19-has-infected-some-570000-health-workersandkilled-2500-americas-paho.

35. Cable News Network (CNN). Over 2,000 health care workers in Ghana have been infected with coronavirus. Updated 1557 GMT (2357 HKT) July 18, 2020. https://edition.cnn.com/2020/07/18/africa/ghana-healthcareworkers-oronavirus/index.htmlon. Accesses 23 June 2021.

36. Kwok KO, Li KK, Wei WI, Tang A, Wong SYS, Lee SS. Editor's choice: influenza vaccine uptake, COVID-19 vaccination intention and vaccine hesitancy among nurses: a survey. Int J Nurs Stud. 2021;114: 103854. https://doi.org/10.1016/j.jinurstu.2020.103854 (Epub 2020 Dec 5).

37. Detoc M, Bruel S, Frappe P, Tardy B, Botelho-Nevers E, Gagneux-Brunon A. Intention to participate in a COVID-19 vaccine clinical trial and to get vaccinated against COVID-19 in France during the pandemic. Vaccine. 2020;38(45):7002-6. https://doi.org/10.1016/j.vaccine.2020.09.041 (Epub 2020 Sep 17).

38. Ditekemena JD, Nkamba DM, Mutwadi A, Mavoko HM, Siewe Fodjo JN, Luhata C, Obimpeh M, Van Hees S, Nachega JB, Colebunders R. COVID-19 vaccine acceptance in the Democratic Republic of Congo: a crosssectional survey. Vaccines (Basel). 2021;9(2):153. https://doi.org/10.3390/ vaccines 9020153.

39. Chew NWS, Cheong C, Kong G, Phua K, Ngiam JN, Tan BYQ, Wang B, Hao F, Tan W, Han X, Tran BX, Hoang MT, Pham HQ, Vu GT, Chen Y, Danuaji R, Rn K, Pv M, Talati K, Ho CS, Sharma AK, Ho RC, Sharma VK. An Asia-Pacific study on healthcare workers' perceptions of, and willingness to receive, the COVID-19 vaccination. Int J Infect Dis. 2021;106:52-60. https://doi. org/10.1016/j.jijid.2021.03.069

40. Afolabi AA, llesanmi OS. Dealing with vaccine hesitancy in Africa: the prospective COVID-19 vaccine context. Pan Afr Med J. 2021. https://doi. org/10.11604/pamj.2021.38.3.27401.

41. Kitonsa J, Kamacooko O, Bahemuka UM, Kibengo F, Kakande A, Wajja A, Ruzagira E. Willingness to participate in COVID-19 vaccine trials; a survey among a population of healthcare workers in Uganda. PloS one. 2021;16(5):e0251992.

42. Enitan SS, Oyekale AO, Akele RY, Olawuyi KA, Olabisi EO, Nwankiti AJ, Enitan CB. Assessment of knowledge, perception and readiness of Nigerians to participate in the COVID-19 vaccine trial. Int J Vaccine Immun. 2020:4(1):1-13.

43. Nzaji MK, Ngombe LK, Mwamba GN, Ndala DBB, Miema JM, Lungoyo $\mathrm{CL}$, Musenga EM. Acceptability of vaccination against COVID-19 among healthcare workers in the Democratic Republic of the Congo. Pragmatic Observ Res. 2020;11:103.

44. Biswas N, Mustapha T, Khubchandani J, Price JH. The nature and extent of COVID-19 vaccination hesitancy in healthcare workers. J Commun Health. 2021. https://doi.org/10.1007/s10900-021-00984-3.

45. Papagiannis D, Rachiotis G, Malli F, Papathanasiou IV, Kotsiou O, Fradelos EC, Giannakopoulos K, Gourgoulianis KI. Acceptability of COVID-19 
vaccination among Greek Health Professionals. Vaccines (Basel). 2021;9(3):200. https://doi.org/10.3390/vaccines9030200.

46. Khairy A, Mahgoob E, Nimir M, Ahmed M, Jubara M, Eltayeb D, Ahmed A. Acceptability of COVID-19 vaccination among healthcare workers in Sudan: a cross sectional survey. 2021; Pre-print: https://doi.org/10.21203/ rs.3.rs-745232/v1.

47. Agyekum MW, Afrifa-Anane GF, Kyei-Arthur F, Addo B. Acceptability of COVID-19 vaccination among health care workers in Ghana. Advances in Public Health, 2021
48. Dror AA, Eisenbach N, Taiber S, Morozov NG, Mizrachi M, Zigron A, Srouj S, Sela E. Vaccine hesitancy: the next challenge in the fight against COVID-19. Eur J Epidemiol. 2020;35(8):775-9. https://doi.org/10.1007/ s10654-020-00671-y (Epub 2020 Aug 12).

\section{Publisher's Note}

Springer Nature remains neutral with regard to jurisdictional claims in published maps and institutional affiliations.
Ready to submit your research? Choose BMC and benefit from:

- fast, convenient online submission

- thorough peer review by experienced researchers in your field

- rapid publication on acceptance

- support for research data, including large and complex data types

- gold Open Access which fosters wider collaboration and increased citations

- maximum visibility for your research: over $100 \mathrm{M}$ website views per year

At BMC, research is always in progress.

Learn more biomedcentral.com/submissions 